\title{
REDIMENSIONANDO EL SIGNIFICADO Y ALCANCE DE LAS COMPETENCIAS PERSONALES
}

RESIZING THE MEANING AND SCOPE OF PERSONAL COMPETENCIES

\author{
Volumen 12, Número 2
}

Mayo-Agosto

pp. 1-28

Este número se publicó el 30 de mayo de 2012

Juan B. Climént Bonilla

Revista indizada en $\underline{\text { REDALYC }}$

Revista distribuida en las bases de datos:

CATÁLOGO DE LATINDEX, IRESIE, CLASE, DIALNET, DOAJ, E-REVIST@S,

Revista registrada en los directorios:

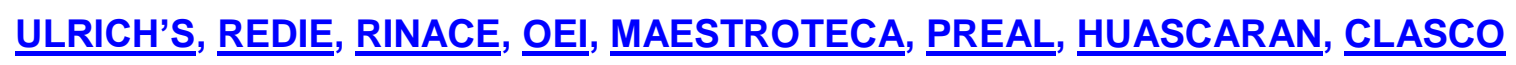




\title{
REDIMENSIONANDO EL SIGNIFICADO Y ALCANCE DE LAS COMPETENCIAS PERSONALES
}

\author{
RESIZING THE MEANING AND SCOPE OF PERSONAL COMPETENCIES
}

\author{
Juan B. Climént Bonilla
}

\begin{abstract}
Resumen: Al paso de tres décadas, con la expansión de la economía global, el movimiento de formación por competencias ha cobrado progresiva influencia en los sectores educativo y laboral de un creciente número de países. Si bien, la literatura especializada abunda en asuntos relacionados con competencias personales, y existe una extensa, aunque relativa, variedad de definiciones sobre lo que éstas son y significan, la noción prevalente de su naturaleza y origen se restringe a marcos conceptuales considerablemente afines. Teniendo en cuenta las implicaciones de esta situación en aspectos teóricos y prácticos concernientes al fomento de las competencias, el presente artículo se adentra en la investigación teórica de sus propiedades, no sólo desde la perspectiva conceptual, sino también, y sobre todo, en el ámbito de la interacción de factores biológicos y no biológicos, que son consustanciales a la naturaleza y el desarrollo de los seres humanos. La investigación se fundamenta en el análisis de dos ejes temáticos: a) la concepción estandarizada de las competencias, y b) el desarrollo de las competencias en relación con factores biológicos y experienciales. De este análisis resulta un racional sobre el papel central de la educación en el desarrollo de las competencias de las personas, pertinente a los avances de la investigación neurocientífica en este campo. Las conclusiones destacan algunas inconsistencias en el fomento de competencias individuales -estandarizadas o no-, asociadas a falsos supuestos u omisiones sobre la naturaleza y formación de los seres humanos a lo largo de la vida.
\end{abstract}

Palabras clave: CAPACITACIÓN, INSTRUCCIÓN, COMPETENCIAS, HABILIDADES, EDUCACIÓN BASADA EN COMPETENCIAS

\begin{abstract}
Through the last three decades, with global economy expansion, the competency-based education and training movement has progressively influenced education and labor sectors of an increasing number of countries. While specialized literature abounds on issues related to personal competencies, and an extensive, although relative variety of definitions exists about them, the prevalent notion of their nature and origin is restricted to considerably similar conceptual frameworks. Taking into account the implications of this situation for theoretical and practical aspects concerning competencies promotion, the present article deepens into the theoretical research of their properties, not only from the conceptual perspective, but also, and mainly, within the scope of biological and non-biological interactive factors, which are consubstantial to the nature and development of human beings. The research is based on the analysis of two thematic approaches: a) the standardized view of competencies, and $b$ ) the development of competencies in relation to biological and experiential factors. From this analysis results a rationale on the central role of education in people's competencies development, pertinent to neuroscience research advances in this field. The findings highlight some inconsistencies in the development of individual competencies -standardized or not-, associated with false assumptions or omissions on the nature and qualitative progress of human beings throughout their life.
\end{abstract}

Key words: TRAINING, INSTRUCTION, COMPETENCES, COMPETENCIES, SKILLS, COMPETENCY-BASED EDUCATION

\footnotetext{
1 Doctor en Educación, por la Universidad de Cornell; ha participado como consultor en diversos estudios y proyectos en materia de desarrollo rural y educación para adultos; desde 1986 es profesor investigador de la Universidad Autónoma Metropolitana, Unidad Xochimilco, en la ciudad de México.
}

Dirección electrónica: jcliment@correo.xoc.uam.mx

Artículo recibido: 4 de mayo, 2011

Aprobado: 12 de abril, 2012 


\section{INTRODUCCIÓN}

Han pasado 37 años desde la publicación del artículo de David McClelland "Pruebas para la competencia en lugar de para la 'inteligencia"' (Testing for competence rather than for "intelligence"); artículo que marcara un hito en la valoración y reconocimiento de las capacidades humanas para resolver problemas y realizar tareas de general y particular interés, en tanto sus argumentos y evidencias tienen fuertes implicaciones en dos sentidos: por una parte, descartan la validez y confiabilidad de las pruebas de "inteligencia" (específicamente del cociente intelectual, $\mathrm{Cl}$ ), entonces en boga, como indicadores fehacientes del desempeño y éxito futuros de las personas; y por otra, suplantan el valor de estos indicadores por el de pruebas de competencia (habilidades laborales) para predecir el desempeño de las personas en el empleo (McClelland, 1973, pp. 4-7).

Más aún, mientras el artículo advierte del sentido discriminatorio y excluyente de la "inteligencia", medida de manera genérica por los convencionales tests, y del poco o ningún valor de los respectivos indicadores para el desarrollo de las personas en el ámbito social y laboral, a la vez arguye sobre las ventajas del carácter abierto e incluyente de los tests de competencia para reconocer y fomentar la eficiencia en el trabajo (prociency on the job) de los individuos.

Hoy día, las variantes cualitativas de la inteligencia parecen haber cobrado mucha mayor importancia e interés que las medidas cuantitativas sobre la misma, como lo muestra la diversificación de sus conceptos: inteligencia social (Thorndike, 1920; Goldman, 2006), inteligencia emocional (Salovey y Mayer, 1989; Goldman, 1995), inteligencias múltiples (Gardner, 1993), e inteligencia práctica (Albrecht, 2008), entre otros.

Por lo que toca a las competencias, al paso de tres décadas se han instaurado un sinnúmero de iniciativas y modelos para la normalización, evaluación, acreditación, reconocimiento y fomento de las "competencias" individuales, en prácticamente todos los ámbitos de la enseñanza y el aprendizaje (formal, no formal e informal), que han trascendido en las políticas y reformas de los sistemas nacionales de educación, de muchos países alrededor del mundo (Coles y Werquin, 2007), sin mencionar la instrumentación de los propios enfoques (orientados a competencias), en los esquemas de desarrollo y competitividad de las organizaciones dentro de los procesos globalizadores.

Sin embargo, en la carrera global por adecuar los sistemas educativos y de capacitación a los cambios y demandas de las llamadas "sociedad del conocimiento" y "era 
de la información", la operacionalización de planes y programas para el fomento de las competencias individuales ha dejado atrás, por mucho, la comprensión de la naturaleza y desarrollo de las competencias mismas, como características inherentes a la naturaleza y circunstancias de los seres humanos. En otras palabras, la creciente corriente en favor de las competencias individuales, contrasta enormemente con el pobre conocimiento que existe sobre sus propiedades y génesis. A partir de esta tesis, y con miras a profundizar en dicho conocimiento, a continuación se examina el significado y alcance de las competencias de las personas, desde dos ángulos de interrelación y análisis: a) la concepción estandarizada de las competencias; y b) el desarrollo de las competencias en relación con factores biológicos y experienciales.

\section{La concepción estandarizada de las competencias}

Es interesante observar que McClelland (1973, p. 7) no le prestó mayor importancia al significado de "competencia" (usando las palabras competence o competency, para el singular, y competencies, para el plural), quizás porque le pareció un tanto obvio. En sentido estricto, le bastó con señalar la relación del término con capacidad adaptativa, habilidades para el trabajo, y eficiencia en el empleo; aunque, en una connotación mucho más amplia, alude a los resultados en el crecimiento de la persona, en cuanto a "experiencia, sabiduría y capacidad para desempeñar eficazmente las diversas tareas que la vida le presenta" (McClelland, 1973, p. 8).

No obstante, al hablar de los tests para la evaluación de la competencia se refiere a éstos en el primer sentido, como pruebas fundamentadas en criterios relativos al desempeño en el empleo (McClelland, 1973, pp. 7-8). Es pertinente hacer notar la clara visión de dicho autor, al advertir la gran sofisticación, tanto teórica como práctica (McClelland, 1973, p. 8), que implica la elaboración y aplicación de estos tests, debido a la notoria complejidad del mundo del trabajo.

Sin embargo, tras dos décadas de la publicación del artículo de McClelland -que diera un giro en los métodos y criterios para evaluar las capacidades individuales asociadas con el éxito en la vida y el trabajo-, y ya avanzada la penetración de políticas, modelos y reformas para impulsar la educación y la capacitación con base en competencias -sobre todo en los países industrializados-, como también la corriente de los procesos de globalización alrededor del mundo, se agudizó, o por lo menos se hizo patente, el debate y la confusión en 
torno al significado y alcance de las competencias de individuos y grupos, debate y confusión que, por cierto, continúan hasta la fecha.

En un intento por contribuir a la definición y clarificación del concepto "competencia", Charles Woodruffe (1993) escribe el artículo “¿Qué se quiere decir por competencia?” (What is meant by a competency?). Entre sus argumentos sostiene, con justa razón, que sin una definición precisa de este concepto:

- La persona que desea especificar las competencias de una organización no tiene una idea clara de lo que busca. No puede emplearse una técnica para identificar competencias [...]

- No puede hacerse una contribución teórica a las causas por las que los individuos tienen o carecen de competencia [competency], ni ofrecerse asesoría con bases teóricas sobre lo que pueda desarrollarse. (Woodruffe, 1993, p. 29)

Por su parte, Woodruffe hace una distinción, un tanto forzada, pero crítica, sobre el significado de "competencia", utilizando dos términos que en inglés son sinónimos, competence y competency, y que, por lo mismo, en español no hay manera de diferenciar.

Así, para dar a entender "qué se quiere decir por competencia", distingue entre:

- Los aspectos del empleo que deben desempeñarse competentemente [considerados como competence]; $y$

- lo que las personas necesitan llevar al empleo para desempeñar dichos aspectos al nivel de competencia requerido [tenidos por competency]. (Woodruffe, 1993, p. 29)

Evidentemente, se trata de dos caras de de la misma moneda: la competencia (competence) definida por los requerimientos de desempeño establecidos por el sector productivo; y la competencia (competency) de las personas para cumplir con estos requerimientos. Sin embargo, el punto central a destacar aquí, es que una cosa son las competencias desde la óptica del mercado laboral -o sea, de la demanda de cualificaciones para la operación y desarrollo de las organizaciones y la economía, en contextos locales, nacionales, regionales o globales-; y otra, lo que representan desde la perspectiva formativa -que aunque lleva implícita la oferta de las cualificaciones adquiridas en el transcurso de la vida por los miembros de una o más poblaciones económicamente activas (PEAs), a partir 
de contextos similares o distintos a los observados en el mercado laboral, no necesariamente se circunscribe ni responde a los requerimientos de éste.

Por consiguiente, no es lo mismo que una persona tenga o no las competencias necesarias para desempeñar determinado papel, puesto o función en el mercado de trabajo, a que disponga $o$ no de cualquier tipo de competencias para ganarse la vida, independientemente de las oportunidades reales.

Ante la persistente necesidad de esclarecer el significado de "competencia" (competency), Hoffman (1999, pp. 275, 282) aduce que las competencias pueden definirse de tantas maneras como soportes lógicos se tengan sobre su uso; lo cual equivale a los usos del propio término en relación con los soportes lógicos en que las competencias se aplican.

Desde este punto de vista, las competencias personales no comparten un solo significado; tienen tantas acepciones como usos se les confieran. En el ámbito de las competencias individuales, Hoffman (1999, p. 283) coincide con Woodruffe (1993, p. 29) al distinguir los aspectos inherentes a los estándares de desempeño, de los aspectos atribuidos al comportamiento humano. Lo que para Woodruffe son competences (requerimientos de desempeño en el empleo), para Hoffman son outputs (estándares de desempeño esperados); lo que para el primero son competencies (patrones de conducta que posee la persona competente), para el segundo son inputs (conocimientos, habilidades y destrezas).

Nuevamente, conviene subrayar, en realidad se trata de una misma noción -la de las competencias estandarizadas- concebida desde dos dimensiones complementarias: la del requerimiento laboral, del lado de la demanda (los empleadores); y la del correspondiente satisfactor, del lado de la oferta (los trabajadores y empleados) para responder a esa demanda.

En el Cuadro №1 aparece el esquema adoptado por la Agencia de Desarrollo de Calificaciones y Currículo, del Reino Unido (Qualifications and Curriculum Development Agency, $Q C D A)$, para estructurar y representar los estándares nacionales de las habilidades claves (también llamadas competencias claves). Como puede observarse, los requerimientos se plantean a partir de dos grandes aspectos: lo que el individuo necesita saber (criterios de competencia) y lo que debe ser capaz de hacer (evidencias de competencia) para satisfacer un estándar. En este contexto, la noción de "competencia personal" asume un sentido bilateral: el normativo, en el ámbito de la cualificación que sirve de referente al desempeño individual "deseado"; y el formativo, en el ámbito del desempeño individual concerniente a la 
cualificación "deseada”. No se puede pensar en un aspecto sin considerar el otro. Ésta es la noción dominante de las competencias personales.

Cuadro № 1. Formato de las habilidades claves, $^{2}$ en Inglaterra.

\begin{tabular}{|l|}
\hline Parte A \\
\hline Necesitas saber cómo... \\
\hline Esta sección expone técnicas y conocimientos \\
asociados con cada competencia clave. Te dice \\
lo que necesitas aprender y practicar para \\
sentirte seguro en la aplicación de estas \\
habilidades en tus estudios, trabajo u otros \\
aspectos de tu vida.
\end{tabular}

\begin{tabular}{|l|}
\hline Parte B \\
\hline Debes... \\
\hline Esta sección se construye sobre la parte A y \\
describe la aplicación de las competencias. \\
Describe las competencias que debes mostrar. \\
Todo tu trabajo para esta sección será \\
evaluado. Debes tener evidencias de que \\
puedes hacer todas las cosas enlistadas en los \\
puntos marcados.
\end{tabular}

Tomado de Qualifications and Curriculum Development Agency, QCDA (2004).

\section{En busca de disyuntivas conceptuales y epistemológicas}

A continuación se presenta, por orden cronológico, una extensa relación de definiciones alusivas al concepto de competencias personales, que proporciona información de interés sobre las propiedades atribuidas a éstas, dentro de un lapso de más de tres décadas (1973-2006):

1. Habilidades de trabajo que determinan un desempeño competente en el empleo (McClelland, 1973, p. 7).

2. "Característica subyacente de una persona que resulta en desempeño efectivo y/o superior en el empleo" (Klemp, 1980; citado por Boyatzis, 1982, p. 21).

3. Característica subyacente de una persona, como por ejemplo: "un motivo, rasgo, habilidad, aspecto de la imagen propia o del papel social, o un cuerpo de conocimiento que él o ella usa [en el trabajo]" (Boyatzis, 1982, p. 21).

4. Habilidad para desempeñar actividades al nivel esperado en el empleo (Lloyd y Cook, 1993, p. 14).

5. Conjunto de patrones -o dimensiones- de conducta que la persona implicada debe llevar a un puesto de trabajo para desempeñar sus tareas y funciones de manera

2 La Agencia para el Desarrollo de Calificaciones y Currículo (Qualifications and Curriculum Development Agency, QCDA), de Inglaterra, utiliza el concepto de key skills (habilidades/destrezas claves), en tanto otras fuentes u organismos, como DeSeCo, en el ámbito de la OCDE, manejan el de key competencies (competencias claves). En inglés es común que ambos conceptos se traslapen o mencionen de manera indistinta, por lo que, al traducirlos al español, la diferenciación es aún menos clara. 
competente -es decir, de acuerdo con los requerimientos fijados; con competencia (competence)- (Woodruffe, 1993, pp. 29, 35).

6. Característica subyacente de un individuo, causalmente relacionada con criterios (estándares) de rendimiento efectivo o superior en una situación o trabajo (Spencer y Spencer, 1993, p. 9).

7. “Capacidad de una persona (o una organización) para alcanzar logros específicos. Las competencias personales comprenden capacidades integradas orientadas al desempeño, que consisten en conjuntos de estructuras de conocimientos, y también de capacidades cognitivas, afectivas y -donde se necesitan-psicomotoras, así como de actitudes y valores, que se requieren para realizar tareas, resolver problemas y, en general, para funcionar eficazmente en cierta profesión, organización, posición o papel” (Mulder, 2001; citado por Biemans et al., 2004, p. 530).

8. Capacidad de satisfacer demandas o llevar a cabo tareas con éxito, constituida de dimensiones cognitivas y no cognitivas (OCDE, 2002, p. 8).

9. Las tareas laborales en que una persona debe ser competente (Dainty, Cheng y Moore, 2005, p. 40).

10. Combinación de conocimientos, capacidades, habilidades, actitudes y valores que se requieren para la comprensión y transformación de una realidad compleja, de entre todo el universo de saberes relacionados con dicha realidad (Mateo, 2006; citado por Mir, 2006).

11. Estándares que especifican el nivel de conocimientos y habilidades necesarios para realizar con éxito, en el mundo laboral, funciones adecuadas para cada grupo ocupacional (Comisión Europea, s.f., a y b).

A partir de las once definiciones expuestas, el Cuadro № 2 destaca, de manera sinóptica, las principales propiedades asociadas con la naturaleza y propósitos de las competencias personales, tanto desde el punto de vista conceptual (explícito), como epistemológico (implícito). La primera columna se refiere al orden cronológico de las definiciones enlistadas (de la primera, en 1973, a la más reciente, en 2006); las columnas 4-8 comprenden propiedades de las competencias en el ámbito conceptual (explícito); la última, por su parte, indica el carácter del soporte lógico (implícito) a que cada definición más se 
apega. De la información recabada en el Cuadro № 2, derivada del análisis de las once definiciones apuntadas, se desprenden las siguientes observaciones:

- Todas las definiciones, salvo la de Dainty et al. ( $\mathrm{N}^{\circ}$ 9), fundamentan las competencias personales en las cualidades del individuo (intelectuales, psicomotoras, afectivas, físicas).

- Sólo la definición de Mulder (№ 7) hace referencia explícita a las competencias personales en el plano colectivo (la organización, en este caso). La atención general de las once definiciones se centra en las competencias personales a nivel individual.

- Prácticamente se omite la noción de las competencias como proceso de desarrollo; no meramente con carácter de resultado.

- La mayoría de las definiciones -salvo las de Boyatzis (№ 3) y Mateo (№ 10)- pone énfasis en el desempeño (usualmente circunscrito al ámbito individual).

- Todas señalan contextos de participación en el trabajo o el empleo, o por lo menos dejan abierta esta posibilidad. En este sentido, cabe pensar, el término "competencia" concierne al trabajo y las relaciones laborales de los individuos dentro de determinado régimen jurídico.

- De tal modo, la gran mayoría de los atributos conceptuales de las competencias personales, en el marco de las definiciones consideradas, se concentra en tres aspectos: a) cualidades individuales, $b$ ) desempeño competente y $c$ ) trabajo/empleo.

- Dentro de los mismos atributos, algunas definiciones se salen del patrón dominante; por ejemplo:

- En lo relativo a otros contextos de participación, las definiciones de Spencer y Spencer ( $N^{\circ}$ 6), Mulder (№ 7), OCDE (№ 8) y Mateo (№ 10) no eximen ambientes de participación distintos al laboral -están abiertas-;

- la definición de Mateo (№ 10) es la única que perfila las competencias hacia la "comprensión y transformación de la realidad", desde una noción sistémica u holista (de la realidad, del universo de saberes, y de la interrelación entre una y otro);

- finalmente, la referencia directa a estándares de competencia se encuentra en las definiciones de Woodruffe (№ 5), Spencer y Spencer (№ 6) y la Comisión Europea (№ 11). 
- Por otra parte, en lo que respecta al soporte epistemológico de las propiedades atribuidas a las competencias personales, cabe destacar dos puntos:

- Nueve definiciones se ajustan al racional del paradigma científico/racionalista: esencialmente analítico, reduccionista, empiricista, asociacionista, reactivista (causa y efecto), nomológico y monista (Guba y Lincoln, 1983, p. 312).

- En contraste con el racional científico/racionalista y, por tanto, con las definiciones concomitantes, sólo la definición de Mateo (No. 10) es congruente con el racional del paradigma sistémico/naturalista: fundamentalmente holista, contextual, inductivo-generalista, multidimensional e interdimensional, informal, natural, humanístico, cualitativo y receptivo-respondiente (Guba y Lincoln, 1983, pp. 313-317).

\section{Cuadro № 2. Propiedades de las competencias personales, de acuerdo con diversas} definiciones.

\begin{tabular}{|c|c|c|c|c|c|c|c|c|c|}
\hline \multirow{4}{*}{ No } & \multirow{4}{*}{ Autor } & \multirow{4}{*}{ Año } & \multicolumn{7}{|c|}{$\begin{array}{c}\text { Propiedades } \\
\text { (Naturaleza y propósitos) }\end{array}$} \\
\hline & & & \multicolumn{6}{|c|}{ Conceptuales } & \multirow{3}{*}{$\begin{array}{c}\text { Epistemológicas } \\
\text { Racional }\end{array}$} \\
\hline & & & \multirow{2}{*}{$\begin{array}{l}\text { Cualidades } \\
\text { individuales }\end{array}$} & \multirow{2}{*}{ Desempeño $^{2}$} & \multicolumn{2}{|c|}{$\begin{array}{l}\text { Contextos de } \\
\text { participación }\end{array}$} & \multirow{2}{*}{$\begin{array}{l}\text { Comprensión y } \\
\text { transformación } \\
\text { de la realidad }^{3}\end{array}$} & \multirow{2}{*}{ Estándares } & \\
\hline & & & & & $\begin{array}{l}\text { Trabajo/ } \\
\text { empleo }\end{array}$ & Otros & & & \\
\hline 1 & McClelland & 1973 & $\checkmark$ & $\checkmark$ & $\checkmark$ & --- & --- & --- & $\mathrm{C} / \mathrm{R}$ \\
\hline 2 & Klemp & 1980 & $\checkmark$ & $\checkmark$ & $\checkmark$ & --- & --- & --- & $\mathrm{C} / \mathrm{R}$ \\
\hline 3 & Boyatzis & 1982 & $\checkmark$ & --- & $\checkmark$ & --- & --- & --- & --- \\
\hline 4 & Lloyd y Cook & 1993 & $\checkmark$ & $\checkmark$ & $\checkmark$ & --- & --- & --- & $\mathrm{C} / \mathrm{R}$ \\
\hline 5 & Woodruffe & 1993 & $\checkmark$ & $\checkmark$ & $\checkmark$ & --- & --- & $\checkmark$ & $\mathrm{C} / \mathrm{R}$ \\
\hline 6 & $\begin{array}{l}\text { Spencer y } \\
\text { Spencer }\end{array}$ & 1993 & $\checkmark$ & $\checkmark$ & $\checkmark$ & $\checkmark$ & --- & $\checkmark$ & $\mathrm{C} / \mathrm{R}$ \\
\hline 7 & Mulder & 2001 & $\checkmark$ & $\checkmark$ & $\checkmark$ & $\checkmark$ & --- & --- & $\mathrm{C} / \mathrm{R}$ \\
\hline 8 & OCDE & 2002 & $\checkmark$ & $\checkmark$ & $\checkmark$ & $\checkmark$ & --- & --- & $\mathrm{C} / \mathrm{R}$ \\
\hline 9 & Dainty et al. & 2005 & --- & $\checkmark$ & $\checkmark$ & --- & --- & --- & $\mathrm{C} / \mathrm{R}$ \\
\hline 10 & Mateo & 2006 & $\checkmark$ & --- & $\checkmark$ & $\checkmark$ & $\checkmark$ & --- & $\mathrm{S} / \mathrm{N}$ \\
\hline 11 & $\begin{array}{l}\text { Comisión } \\
\text { Europea }\end{array}$ & $s / f$ & $\checkmark$ & $\checkmark$ & $\checkmark$ & --- & --- & $\checkmark$ & $\mathrm{C} / \mathrm{R}$ \\
\hline
\end{tabular}

C/R Científico/Racionalista S/N Sistémico/Naturalista

1 Capacidades, conocimientos, habilidades, valores, comportamiento.

2 Eficacia, eficiencia, efectividad, logro (principalmente en torno a expectativas de terceros).

3 En el sentido más amplio (político, social, cultural, económico, medio ambiental) 
Si, como señala Hoffman (1999, pp. 275, 282), las competencias pueden definirse de tantas maneras como soportes lógicos se tengan sobre su uso, y por tanto, el término "competencia" debe usarse de modo que refleje el propósito y sentido de su aplicación, es por demás notorio que entre los significados de las definiciones apuntadas destaca el énfasis en el rendimiento en el trabajo, al que son inherentes variables de índole económica relevantes a la producción de bienes y servicios.

En esta lógica, cabría afirmar que tanto los usos y propósitos de las "competencias" personales, como el significado de sus definiciones, están sujetos a los preceptos del paradigma de investigación y desarrollo a que son afines -por comunión y soporte recíproco-. Es aquí donde radica el trasfondo de la naturaleza y propósito de las competencias personales, vistas desde la óptica de la concepción estandarizada.

\section{El desarrollo de las competencias en relación con factores biológicos y experienciales}

Por complejos que parezcan los acercamientos estandarizados a las competencias referentes de desempeño esperado (criterios y evidencias de competencia), instrumentos de evaluación (normas o estándares), e incluso, sistemas nacionales de calificaciones-, su conocimiento y comprensión es mucho más simple que el que actualmente entraña el origen y desarrollo de éstas en los seres humanos. Si bien, las cualidades individuales (intelectuales, psicomotoras, afectivas y físicas) pueden adquirir infinidad de patrones en la conformación y despliegue de las competencias que poseen las personas, todas las competencias, independientemente de su tipo, nivel y contexto, se originan -estructural y funcionalmente- en el cerebro.

Desde esta perspectiva, pudiera considerarse que las competencias personales parten de las cualidades instrumentales del cerebro, o sea, de las cualidades que permiten al individuo estructurar funcionalmente las distintas características y atributos de que dispone (incluyendo las capacidades cerebrales) para adaptarse al medio y salir adelante en la vida.

En consecuencia, lo que le suceda al cerebro, biológica y experiencialmente, a través del lapso de vida (de la concepción a la muerte del individuo), habrá de afectar en alguna forma y grado las capacidades del propio cerebro, en interrelación estrecha con el desempeño de funciones y roles que comprometen una o más de las competencias 
personales. Estos planteamientos se apoyan en hallazgos de la neurociencia que conciernen al aprendizaje desde temprana edad, como se verá a continuación.

\section{Aprendizaje y plasticidad cerebral}

El desarrollo de las células del sistema nervioso (neuronas) comienza en el embrión, poco después de la concepción, y continua de forma sorprendente, al punto que al cuarto mes de gestación, el número de neuronas alcanza alrededor de 200 billones, aunque cerca de la mitad morirá en el transcurso del quinto mes, como parte del proceso natural del desarrollo fetal (Sousa, 2006, p. 24). El sistema nervioso tiene la capacidad de aumentar o disminuir el número de ramificaciones y conexiones neuronales, según lo requiera. Esta capacidad, conocida como plasticidad cerebral o neuroplasticidad, es enorme durante los primeros meses o años de vida (Acarín y Acarín, 2005, p. 203).

Desde que el niño ${ }^{3}$ nace, conforme recibe estímulos del medio ambiente, el cerebro desarrolla conexiones neuronales (sinapsis) a un paso increíble. Entre más rico sea el entorno, mayor será el número de interconexiones formado y, por consiguiente, el aprendizaje puede ser más rápido y tener mayor significado; así, desde temprana edad, las experiencias están ya conformando el cerebro y diseñando la arquitectura neuronal única que influirá en la manera de bregar con futuras experiencias en la escuela, el trabajo y otros lugares (Sousa, 2006, p. 24). Conforme el niño se aproxima a la pubertad, la plasticidad cerebral se estabiliza y dos procesos comienzan: por una parte, las conexiones que el cerebro encuentra útiles se vuelven permanentes, y por otra, las que no lo son se eliminan; de esta manera, con base en la experiencia, el cerebro fortalece y desecha selectivamente las conexiones neuronales (Sousa, 2006, p. 24).

Aunque a partir de los 20-25 años la capacidad neuroplástica empieza a declinar, con entrenamiento apropiado las personas pueden mantenerla hasta edades avanzadas, de modo que el aprendizaje puede extenderse a prácticamente todo el lapso de vida, desde el nacimiento hasta los 80 años o más (Acarín y Acarín, 2005, p. 203).

El estudio de la organización sistémica y neuronal de la actividad nerviosa superior muestra que la base funcional del proceso asociativo es la formación durante el aprendizaje

3 En el texto de este artículo, el masculino gramatical relativo a niño o niños lo mismo se emplea para referirse a individuos de sexo masculino que a individuos de sexo femenino (niñas); en otras palabras, el sustantivo niño $o$ niños se utiliza para referirse a los infantes, en general, sin distinción de sexos. 
de un estado cualitativamente específico del cerebro que determina conductas reflejas condicionadas, y que cada conexión temporal es resultado de la adquisición del cerebro de un nuevo estado a partir de mecanismos de plasticidad; asimismo, a la multiplicidad de estados del cerebro corresponde una diversidad de combinaciones de estímulos, motivaciones, refuerzos y reacciones en un individuo (Kotlyar, 1992, p. 267). Se infiere que dentro de esta organización sistémica y neuronal, en estrecha interacción con el medio ambiente, se gestan las cualidades del cerebro que comandan al conjunto de patrones o dimensiones de conducta, llamado por Woodruffe competencias (competencies).

Si consideramos que es el potencial de plasticidad de las relativamente estereotipadas unidades (neuronas) del sistema nervioso, lo que a cada uno de nosotros dota de individualidad (Kandel, Schwartz y Jessell, 2000, p. 34), las cualidades del cerebro tienen una génesis única, absolutamente opuesta a la idea de las competencias estandarizadas.

\section{Periodos críticos y "competencias" tempranas}

Al nacimiento, el cerebro del ser humano está listo para enviar señales a otras partes del cuerpo para formar conexiones o sinapsis. Si las sinapsis no se forman durante el tiempo apropiado (periodo crítico o ventana de oportunidad), el potencial de conexión se pierde, al punto que la falta de estimulación puede provocar que las neuronas mueran o se desvíen a otra función (lowa State University Extension, 2006).

Las "ventanas de oportunidad" son lapsos de tiempo en que el niño puede aprender y desarrollar habilidades mejor. Después de esos lapsos el aprendizaje suele ser más difícil, requiere mayor esfuerzo y práctica, y los resultados no son tan buenos (lowa State University Extension, 2006). Durante los periodos críticos los sistemas y mapas del cerebro se desarrollan potencialmente con ayuda de los estímulos que recibimos de nuestro entorno (Doidge, 2007, p. 99). Fuera del antro materno, los dos primeros años de vida son especialmente críticos:

el cerebro promedio, de pesar 400 gramos al nacimiento, alcanza 1,000 gramos en 12 meses. Somos tan dependientes del amor y los cuidados de otros, en parte porque grandes áreas de nuestros cerebros no empiezan a desarrollarse sino hasta después de que nacemos. Las neuronas en el cortex prefrontal, que nos ayuda a regular nuestras emociones, hacen conexiones en los primeros dos años de vida, pero esto 
sólo ocurre con ayuda de otras personas, que en la mayoría de los casos es la madre, quien literalmente moldea el cerebro de su bebé. (Doidge, 2007, pp. 343-344)

Hoy se sabe que los niños nacen con ciertas capacidades biológicas para aprender (reconocen sonidos, distinguen objetos animados de inanimados, y poseen un sentido inherente del espacio, el movimiento, el número y la causalidad), y que estas capacidades incipientes son activadas por el medio ambiente, de modo que los procesos de desarrollo implican interacciones entre las "competencias tempranas" (early competencies) de los niños, y el medio ambiente y los apoyos interpersonales; así, tales interacciones, y particularmente los apoyos interpersonales, fortalecen las capacidades que son relevantes a los entornos del niño y desechan las que no lo son (Bransford, Brown y Cocking, 2000, p. 234). A este respecto, es de suma importancia tener presente, primero, que dichas capacidades biológicas -entre otras cualidades intelectuales y físicas de los niñosrepresentan estadios incipientes o tempranos de la parte biológica de posibles competencias personales futuras; y segundo, que las competencias personales, como tales, implican procesos de desarrollo y formación integrales (entre aspectos biológicos y formativos). En los bebés, y los niños en general, las cualidades cerebrales son fundamentalmente de carácter biológico, no formativo; conforme avanza la edad, en la medida en que esas cualidades remontan y se definen, a través de la experiencia (interior y con el medio), es que determinadas competencias se logran.

En ocasiones las habilidades físicas e intelectuales y los intereses y motivaciones advertidos durante la infancia (por el propio niño o por otras personas), llegan a ser parte de las futuras competencias del individuo; sin embargo, comúnmente emergen otras habilidades y valores, de distinto tipo y magnitud, a través de la vida y en la interacción con diferentes entornos (personal, social, cultural, laboral, ambiental). De tal forma, el conjunto de competencias que posee el adulto para funcionar y ser útil, de manera autosuficiente, en la sociedad (local, nacional, regional, internacional, global), suele ser incierto en etapas tempranas de la vida. Lamentablemente, en nuestros días, el afán administrativo por la evaluación de resultados, más que de procesos (Mott y Granata, 2006, pp. 49-50), parece haber tergiversado el sentido de las competencias en el ámbito educativo.

El absurdo de las pruebas de inteligencia en los niños para predecir desempeño laboral en los adultos, señalado por McClelland, ha sido reemplazado por otro absurdo: aplicar 
pruebas para "evaluar las competencias de los educandos" (Climént, 2010, p. 2), sin una amplia perspectiva de lo que estas son y representan, y mucho menos de los procesos y condiciones necesarios para su desarrollo adecuado. Esta medida, además de que no aporta información diagnóstica y correctiva de fondo, se desvincula del apoyo oportuno y efectivo a las capacidades y procesos de aprendizaje que requieren de especial atención (entornos apropiados) durante los periodos críticos de diversas habilidades, como: desarrollo motor, control emocional, vocabulario, lenguaje hablado, pensamiento lógico, música, cognición espacial, imaginación y creatividad, entre otras (Smith, 2002, 2008; Sousa, 2006, p. 25).

Es importante tener presente que las políticas y programas de educación de un país, especialmente a nivel básico (incluyendo preescolar), deben fomentar las habilidades críticas del aprendizaje, en relación con los periodos críticos y entornos apropiados correspondientes -de índole general y particular-, a sabiendas de que el dominio efectivo de las habilidades críticas (claves, centrales, nucleares, funcionales) impacta directamente la calidad, diversidad y nivel de las competencias individuales y colectivas (estandarizadas o no), relevantes a la formación y las actividades sociales y económicas de las personas adultas. De no hacerse así, es de esperar que el balance de competencias de la PEA disminuya considerablemente sus posibilidades de mejorar, se mantenga bajo o empeore, en menoscabo de la calidad de vida de los ciudadanos (Finegold y Soskice, 1991; citados por Hodkinson, Sparkes y Hodkinson, 1996, p. 9).

\section{El papel de la experiencia en el desarrollo de las competencias personales}

Si bien las propiedades anatómicas y fisiológicas del cerebro quedan establecidas a partir de la dotación genética, en el periodo prenatal y los primeros 20 años de vida, la experiencia ejerce un poderoso papel en el proceso de desarrollo y el despliegue de capacidades de este órgano; asimismo, es preciso entender, la experiencia no proviene del entorno per se, sino de la compleja interacción bidireccional entre el medio ambiente y el cerebro en desarrollo (Nelson, de Haan y Thomas, 2006, p. 30). Básicamente, pues, las competencias personales son resultado de la estrecha interacción entre la parte biológica (nature) y la parte experiencial (nurture) del ser humano. Mientras los principios rectores fundamentales de la biología -y por tanto, de la parte biológica de las competencias- se encuentran en los genes (Goldberg y Weinberger, 2009, p. 3), los diversos factores (sociales, culturales, económicos, físicos) con que el cerebro interactúa y adquiere experiencia 
(competencias) se encuentran en el medio ambiente. Desde esta lógica -siguiendo a Goldberg y Weinberger (2009, p. 16)- las competencias pueden considerarse fenotipos complejos ("características o cualidades complejas") determinados por múltiples variables (biológicas y no biológicas).

El genoma humano, conformado por un total de 20,000 a 25,000 genes (International Human Genome Sequencing Consortium, 2004, p. 931), contiene la información básica necesaria para el desarrollo de las características físicas e intelectuales del individuo, hasta su límite biológico. Así, la variación en el desempeño de cierta competencia (fenotipo complejo) entre las personas que la poseen, puede explicarse por la variación de factores genéticos (biológicos), por la variación de factores ambientales (no biológicos), y por la variación de la interacción de unos con otros (experiencia). Aunque la dotación genética para una competencia (fenotipo complejo) varía entre individuos, para un ser humano es prácticamente la misma, desde el nacimiento hasta la muerte. De ahí que, lo que podemos hacer para mejorar una o más de las competencias personales (propias y de otros) depende fundamentalmente de los progresos que consigamos -circunstancial o deliberadamente- en dos conceptos estrechamente asociados: el entorno de aprendizaje y la experiencia individual.

En materia de desarrollo cerebral y neuroplasticidad, existe evidencia de que, en tanto la sobreproducción sináptica (más conexiones neuronales de las necesarias) es obra principalmente del control genético, la reducción de sinapsis hasta un número presumiblemente óptimo, depende en parte de la comunicación entre neuronas (Nelson et al., 2006, p. 25). La Figura № 1 presenta un esquema de la evolución sináptica, de un caso hipotético, donde destaca la interrelación de factores biológicos, medioambientales y experienciales sobre el curso de procesos de sobreproducción y reducción de conexiones neuronales de la corteza cerebral. La curva superior (línea continua) describe la cantidad de sinapsis biológicamente posible, sobre un referente máximo de 100 por ciento, en el curso del lapso de vida.

En contraparte, la curva inferior (línea intermitente) muestra la cantidad de conexiones que en realidad el cerebro alcanzó a lo largo de dicho lapso. La curva superior (potencial) no puede ser mayor; la inferior (real), en cambio, tiene la posibilidad de acercarse significativamente a la superior, pero también de distanciarse hasta grado extremo. Entre ambas curvas se abre el espacio potencial de la capacidad biológica, y en este espacio 
interactúan factores genéticos, medioambientales y experienciales. De un entorno rico puede esperarse un mayor número de experiencias enriquecedoras (con propiedades de alto valor), que contribuya a cerrar la distancia entre la curva real y la potencial; el entorno pobre, por el contrario, en tanto dificulta u obstaculiza este acercamiento (aporta poco o ningún valor a las propiedades de la experiencia), tiende a abrir la brecha.

Como se señaló -a partir de un ejemplo hipotético-, cada curva describe la cantidad relativa de sinapsis, con respecto a un nivel máximo (100 por ciento), considerando el cortex cerebral completo. En otros modelos pudieran analizarse las curvas de sinapsis de una 0 más de las áreas específicas de este órgano -como el cortex frontal, parietal, temporal $u$ occipital-, en relación con funciones o habilidades concretas y, si fuera el caso, con determinados precursores o componentes de competencias personales.

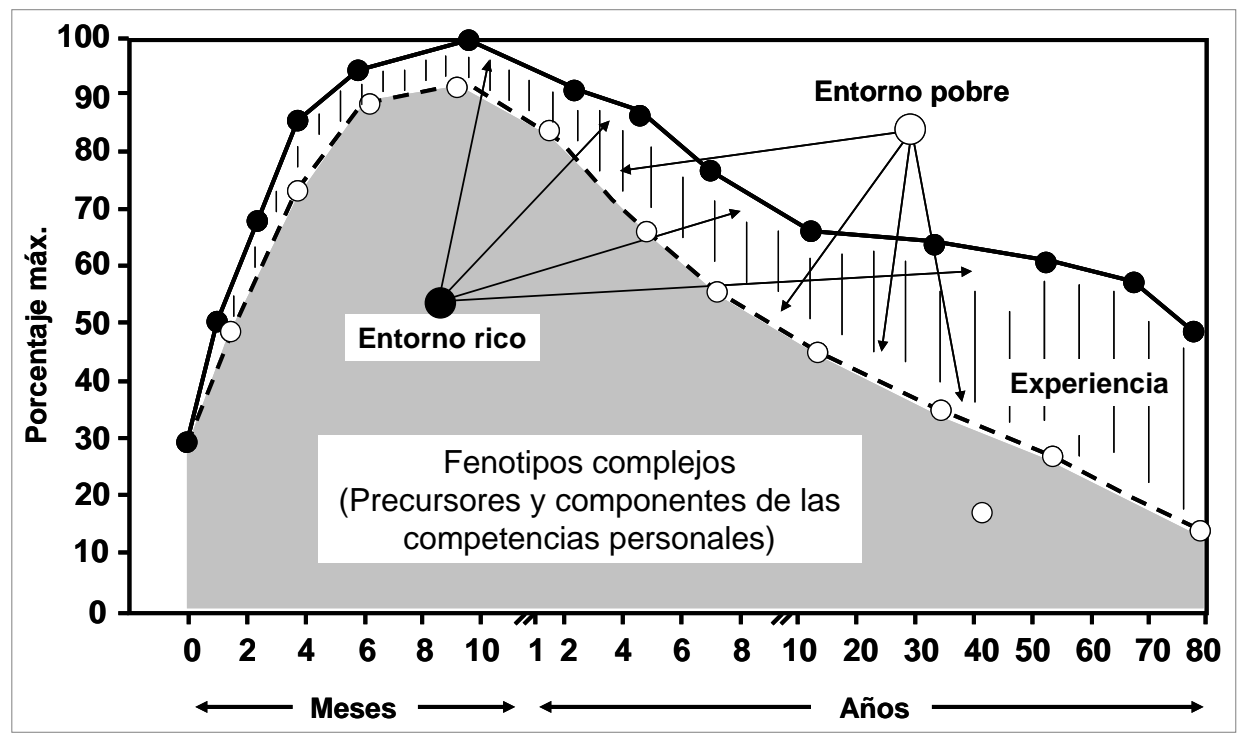

Figura № 1. El papel de la experiencia en el desarrollo sináptico del cerebro. Adaptado de Huttenlocher (1994), citado por Nelson et al. (2006, p. 26).

Según se aprecia en la Figura № 1, los precursores y componentes de las competencias personales (fenotipos complejos) están acotados por la curva efectiva (real) del desarrollo sináptico, dentro del área sombreada. En la medida en que el entorno de aprendizaje y los eventos experienciales son mejores, esta área puede expandirse hacia los linderos del potencial genético (curva superior), incrementando la cantidad y calidad de los precursores y componentes de las competencias mismas, durante el proceso de formación. 


\section{Disección de las competencias personales}

En general, entre la comprensión de las competencias y la definición de su significado independientemente de la versión de que se trate-, existe un contrasentido fundamental: por un lado, las múltiples y variadas propiedades de las competencias, sin considerar la infinidad de formas y contextos en que pueden estructurarse y manifestarse, hacen de ellas entidades conceptuales en extremo complejas; por otro, la definición del término "competencia", como referente claro, común y unívoco, supone la sobresimplificación, y usualmente el sesgo, en la ponderación de dichas propiedades. Sin embargo, en lo particular, ambos ejercicios -el de comprensión y el de definición- mantienen estrechos lazos de reciprocidad entre y con sus respectivos andamiajes; de ahí la necesidad de profundizar en el conocimiento de los elementos que sirven, o pueden servir, a manera de plataforma común, para el desarrollo y fomento de las competencias.

Del análisis realizado en los apartados anteriores, se desprende que la comprensión y significado de las competencias personales implica tres grandes esferas:

a) Situación deseada. Atañe a lo que una o más personas adultas -tenidas por tales, desde el punto de vista jurídico, social, económico y psicológico- deben ser capaces de hacer, conseguir o lograr, con respecto a expectativas propias y/o de terceros, bajo cierta concepción de mérito o valor, estandarizada o no.

b) Proceso de desarrollo. Concierne a las etapas, precursores y componentes de la formación de competencias inherentes a la preparación de las personas (en su actual o futura condición de adultos), en relación con la situación deseada y el contexto de formación.

c) Contexto de formación. Asociado al carácter y desarrollo de la experiencia, individual y colectiva, a través de la interacción de múltiples y diversos factores, de orden biológico y no biológico, conforme avanza la edad y madura el cerebro.

Con el fin de ahondar en el origen y naturaleza de las competencias, a continuación procederemos a su "disección" en el espacio de cada una de las tres esferas mencionadas. Para cada caso se utilizará, como ejemplo, un esquema que ilustre la "disección" desde la perspectiva correspondiente: el mapa funcional, para el examen de las competencias en el ámbito de la "situación deseada"; una habilidad funcional, comprensión de lectura, concerniente al "proceso de desarrollo" de competencias futuras; y el concepto de 
experiencia, consustancial a la interiorización del "contexto (político, social, cultural, laboral, económico, ambiental) de formación" para la vida y el trabajo.

\section{Mapa funcional (Situación deseada)}

Consiste en la representación gráfica del conjunto estructurado de funciones, roles, unidades y elementos de competencia, para el establecimiento de estándares o normas acordes, en una organización o actividad productiva. Como se aprecia en la Figura № 2, el propósito principal, equivalente a la función medular de una organización o actividad productiva, se desagrega en papeles claves; estos, en funciones claves; las funciones claves, en unidades de competencia; y éstas, a su vez, en elementos de competencia.

Estos últimos, finalmente, describen los criterios de desempeño -lo que una persona debe ser capaz de hacer- y las evidencias de conocimiento, producto y desempeño -las pruebas para demostrarlo- (Lloyd y Cook, 1993, pp. 43, 70-73). Aunque los niveles de desagregación varían en número y tamaño, según la complejidad del propósito o función principal, el desglose comúnmente adopta un formato igual o similar al del esquema mostrado en la Figura № 2. Así, las funciones que son eje de las organizaciones y actividades productivas de una economía se traducen en requerimientos de desempeño (competencias) vinculados a indicadores de eficiencia y competitividad. En esta esfera se conforma la imagen dominante de las competencias, que las refiere como estándares o expectativas de desempeño deseado (competente).

Es conveniente advertir que los elementos de competencia, en calidad de componentes básicos de las normas o estándares del propio orden, no tienen carácter de competencias; más aún, la certificación de las competencias de un individuo se otorga a nivel de unidad (considerando todos los elementos de esta) o de calificación (abarcando todas las unidades de la norma o estándar), pero no a nivel de elemento.

Por lo tanto, no debe confundirse la condición de componente, y mucho menos la de precursor de una o más competencias, con la de competencia. Esta distinción es importante, de manera particular y conjunta, en lo concerniente a las tres esferas descritas. 


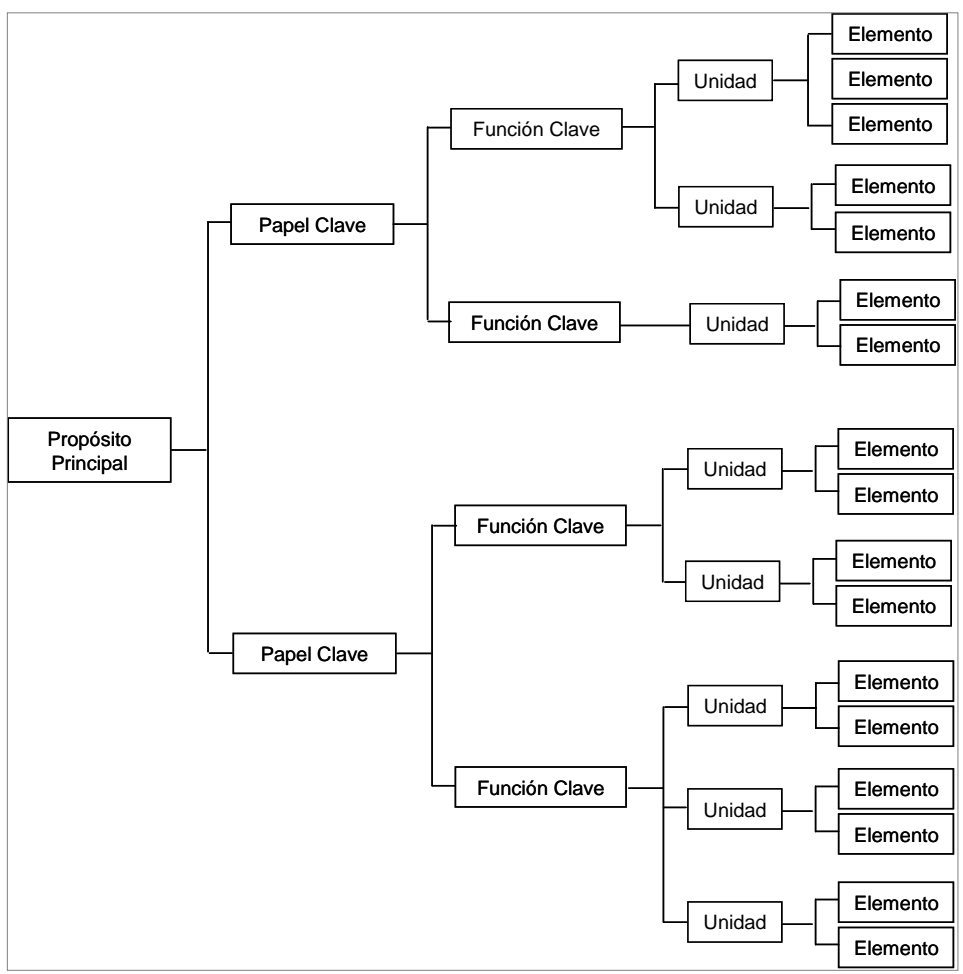

Figura № 2. Estructura convencional del mapa funcional. Tomado de Lloyd y Cook (1993, p. 71).

Entre los elementos de competencia -la parte fina de las normas o estándares de evaluación- y las facultades físicas y mentales del individuo, está el conjunto de conocimientos, habilidades y valores (entre otras posibles cualidades) que el propio individuo posee, o debe poseer, para ser sujeto de acreditación y reconocimiento, bajo los criterios y procedimientos del sistema de certificación establecido.

Sin embargo, la concepción de este conjunto es distinta, según la cara de las competencias por donde se mira: mientras del lado de la norma o estándar de competencia se refiere a resultados y productos esperados (es superficial o plano); del lado del individuo supone etapas y procesos de formación (tiene fondo). Puede decirse que la primera concepción proporciona una imagen reduccionista de la segunda, puesto que las competencias, por naturaleza, son muy complejas.

Las competencias de las personas, vistas como estándares de evaluación, no tienen más componentes que los elementos de competencia (básicamente, criterios y evidencias de desempeño); en cambio, como fenotipos complejos implican una serie de precursores y 
componentes (conocimientos, habilidades, valores, actitudes, atributos personales), de distinto tipo y grado de sofisticación -según la competencia de que se trate-, que a su vez, y de manera sucesiva, tienen sus respectivos precursores y componentes, hasta alcanzar los principios y mecanismos fundamentales del aprendizaje y la conducta. De ahí que sea un error llamar "competencias" a las expresiones de desempeño (conocimientos, habilidades, valores, actitudes), estandarizadas 0 no, que en realidad constituyen precursores 0 componentes de éstas.

Por ejemplo, en el Reino Unido se considera actualmente que las habilidades funcionales (functional skills) -específicamente, lenguaje, matemáticas y TIC- proporcionan a las personas las habilidades necesarias para desenvolverse con seguridad y de manera efectiva e independiente en el aprendizaje, la vida y el trabajo (Department for Children, Schools and Families, s.f., p. 2). Estas habilidades son precursoras y componentes de las competencias para la vida y el trabajo, pero en sí mismas no constituyen competencias -no en los adultos, mucho menos en los niños-. Además, las habilidades funcionales -como comprensión de lectura, esquematizada en la Figura № 3- tienen sus propios precursores y componentes, que guardan, o habrán de guardar, su correspondiente lugar en la estructura de fenotipos complejos de competencias individuales y colectivas.

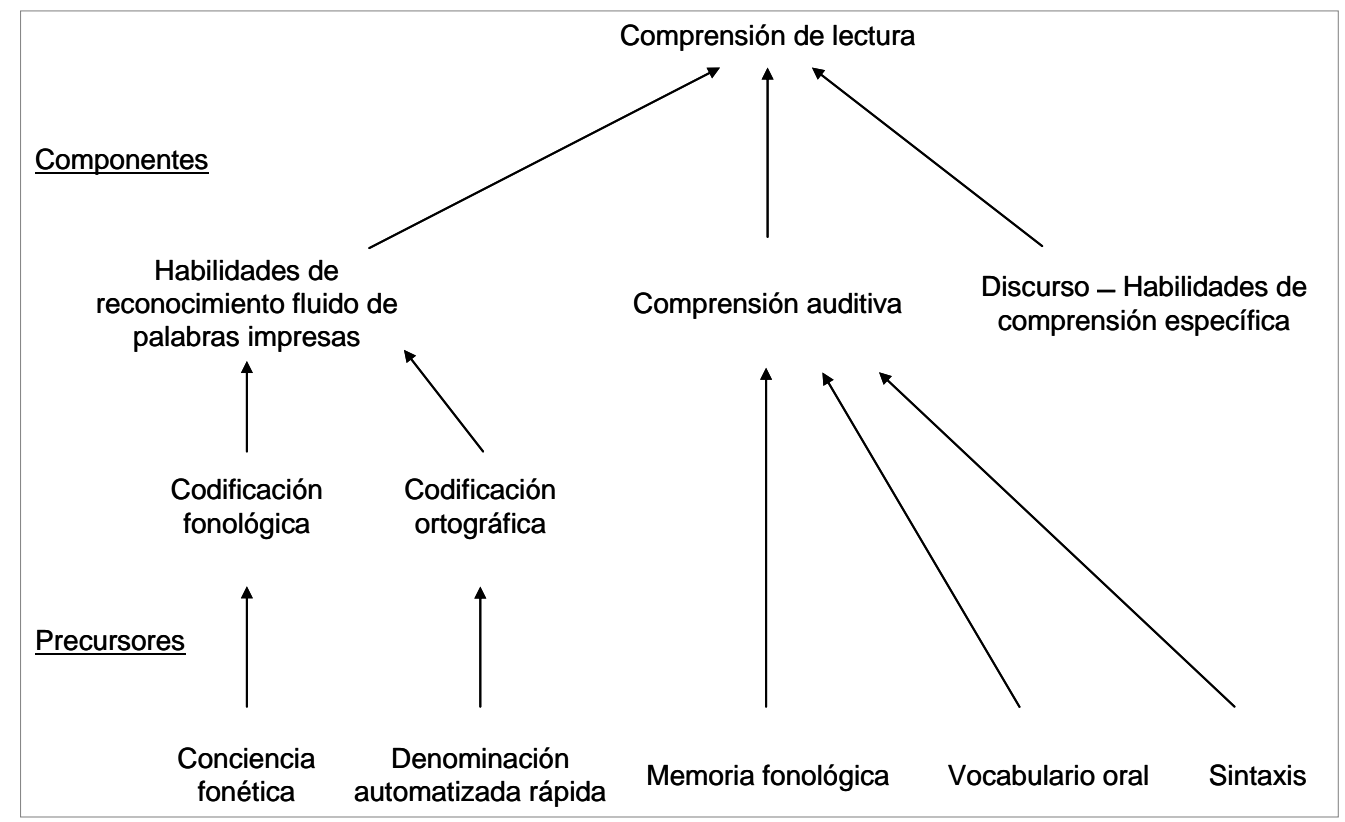

Figura № 3. Factores que impactan la comprensión de lectura. Tomado de Goldberg y Weinberger (2009, p. 178). 
Adviértase que la habilidad de comprensión de lectura es una función cognitiva que, como tal, mantiene estrecha conexión con las bases neuronales del desarrollo del habla y el lenguaje, dominio donde el papel de la experiencia en el desarrollo del cerebro y la conducta ejerce una clara y notoria influencia (Nelson et al., 2006, pp. 58-59).

\section{Experiencia (Contexto de formación)}

Diversos estudios demuestran el poderoso papel del medio ambiente en la regulación y mediación de los efectos genéticos en la neuroplasticidad y la conducta, en el cerebro en desarrollo (primeros 20 años de vida) y en el cerebro desarrollado (adulto), así como en distintas propiedades cerebrales que difieren dramáticamente en y entre individuos (Nelson et al., 2006, pp. 31-33).

Desde un punto de vista científico, el análisis de la experiencia -entendida como la interacción del cerebro con el medio ambiente- debe atender la descripción de las circunstancias y antecedentes, la etapa de desarrollo, el estadio de madurez cerebral, y la experiencia específica a que el individuo es expuesto (Nelson et al., 2006, p. 32) (véase la Figura № 4). Estos aspectos son centrales al contexto de formación de las competencias personales (desde sus bases neuronales y precursores, hasta sus componentes y formas preponderantes) y, por ende, a la complementación de las dos posturas convencionales para definirlas, analizarlas y fomentarlas: la "situación deseada", el lado (evaluativo) de la expectativa de desempeño; y el "proceso de desarrollo", el lado (formativo) del individuo y sus capacidades. 


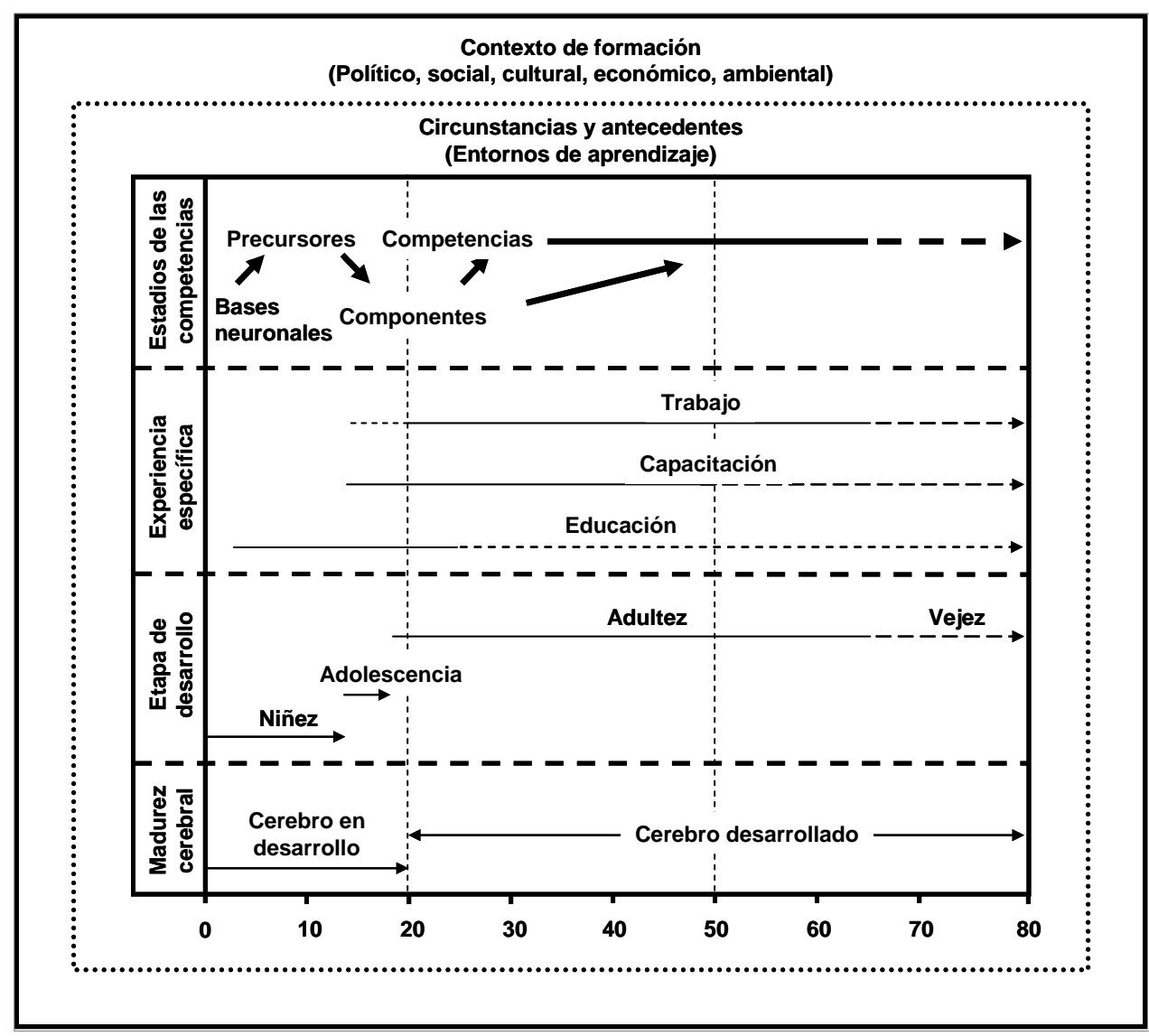

Figura № 4. Contextualización y ejes fundamentales de la formación de competencias.

Los estándares o normas de evaluación hacen referencia, en el mejor de los casos, al medio operativo de las competencias, pero normalmente omiten otro tipo de contextos (político, social, cultural, económico, ambiental); consecuentemente, también pasan por alto los valores, principios o pautas que, más allá del ámbito técnico, marcan la conducta humana, confieren sentido a los actos y son determinantes en el curso que toma la vida. Las competencias tienden a desvirtuarse en la medida en que carecen de principios y valores, al punto que pueden dejar de serlo y convertirse en pseudo o anti-competencias, al margen o contrarias al sentido del bien común (Climént, 2009, pp. 15-16). Por lo tanto, la conciencia de contextos -y los correspondientes valores- que se adquiere a través de la vida, conforme las personas maduran, es esencial para la formación de las competencias, a nivel individual y colectivo. 
De manera gradual, conforme el cerebro se desarrolla y la vida avanza, se definen los intereses y la vocación de las personas, que eventualmente comprometen a las neuronas en determinadas funciones sociales y económicas. Sin la debida conciencia de los contextos y valores pertinentes, y en tanto tales funciones no estén bien establecidas, los fenotipos de las personas, por complejos que sean, no tienen categoría de competencias, es decir, en el plano de la formación y la experiencia, carecen de la madurez y los componentes esenciales para ser considerados competencias.

\section{CONCLUSIONES}

Las pruebas de competencia laboral -basadas en criterios y evidencias de desempeñoen la lógica de que constituyen mejores predictores de eficiencia en el trabajo que los tests de cociente intelectual, no sólo sustituyeron a éstos como instrumentos de evaluación; además, se convirtieron en elementos rectores de políticas e iniciativas de reforma a diversos sistemas nacionales de educación y capacitación, en la economía global.

A las presiones y demandas de los acelerados procesos globalizadores, el movimiento de formación por competencias, suscitado en las últimas tres décadas, ha respondido con el establecimiento de sistemas de calificaciones estandarizadas que impactan la orientación y reconocimiento del aprendizaje, tanto en el ámbito educativo como en el laboral; sin embargo, en el énfasis por la evaluación y obtención de resultados, el propio movimiento perdió de vista el origen y desarrollo de las competencias que promueve, en relación con factores biológicos y experienciales.

A falta de este conocimiento, y bajo la fuerte influencia de la noción estandarizada de las competencias en los sistemas de formación, los soportes lógicos del enfoque de competencias suelen sustentarse, de manera explícita o velada, en los principios y propósitos del crecimiento económico y el paradigma científico dominante, sin reparar en las limitaciones y contrasentidos (reduccionismo, fragmentación, descontextualización) que este sesgo y dicha omisión pueden generar.

En la misma corriente, las acepciones más comunes de las competencias personales se circunscriben a los preceptos del paradigma científico-racionalista; así, en el conjunto de las definiciones aquí analizadas, destacan tres aspectos de las competencias estrechamente ligados con la actividad productiva: a) las cualidades individuales, b) el desempeño competente, y c) el trabajo/empleo. 
El hecho de que las competencias comúnmente se acojan a los valores y premisas de determinado paradigma (científico, tecnológico, político, económico), no significa que sean exclusivas de éste. Las personas pueden valerse de sus competencias para adaptarse a paradigmas de distinta índole, pero también para crear nuevos, o recrear los ya existentes. Pese a estas posibilidades, el soporte lógico de las competencias individuales normalmente se apoya en los axiomas básicos de un paradigma específico, ya establecido, al que retroalimentan (como lo constata el examen de las definiciones de competencia referidas en este artículo).

En la concepción teórica de las competencias, y las subsecuentes implicaciones prácticas, predomina la noción de referentes o instrumentos de evaluación, desde dos perspectivas: la de los requerimientos o expectativas de desempeño individual (estandarizados o no), establecidos por el propio individuo o por terceros; y la de las capacidades de uno o más individuos, en relación con los requerimientos o expectativas fijados. Sin embargo, la interconexión entre ambas perspectivas, pese a ser condición sine qua non para el desarrollo y fomento de las competencias, usualmente pasa inadvertida, de modo que se soslaya o minimiza la importancia de la dimensión formativa (gradual, contextual y experiencial), tanto en la consecución de las competencias como en las cualidades de éstas.

El análisis pormenorizado de las competencias individuales genera información muy distinta, cuando se realiza del lado evaluativo, vinculado a la demanda, que cuando se plantea del lado del individuo, concerniente al proceso de desarrollo personal. Conocida es la complejidad que supone la desagregación de las competencias, en calidad de estándares o instrumentos evaluativos -de los principales propósitos de funciones o actividades de carácter económico o profesional, a unidades y elementos de competencia-, pero mucho más compleja resulta -en sentido opuesto- la integración gradual de las competencias en el ámbito formativo -desde las bases neuronales y los necesarios precursores, hasta sus componentes y formas preponderantes-, cuando se ciñe a fenotipos complejos (características o cualidades individuales complejas) de cierto mérito, valor o sentido, sujetos a la influencia y los efectos de un sinnúmero de variables biológicas y no biológicas.

Entre las dos facetas fundamentales de las competencias -la de lo que se pretende o desea, y la de lo que se consigue o logra-, la experiencia desempeña un papel clave, al procurar la complementación de ambas, a través de la madurez cerebral, las etapas de 
desarrollo, las situaciones específicas, y los estadios de las propias competencias, en el curso de la vida de cada individuo. Todavía más importante, dentro de dicho papel, la experiencia permite el aprendizaje de valores inherentes al contexto de formación, que es determinante en el carácter y sentido de las competencias individuales y colectivas.

Mientras la formación por competencias está dirigida a y por estándares preestablecidos -comúnmente referentes de mérito o valor en actividades productivas-, el desarrollo de bases, precursores y componentes de futuras competencias puede apegarse a esos estándares, lo mismo que mantenerse al margen de ellos, según corresponda a la etapa, las necesidades y el entorno de formación de cada individuo.

De poco o nada sirven las pruebas de evaluación de competencias, cuando en el medio donde las personas viven prevalecen condiciones sociales y económicas desfavorables (como atraso, pobreza, desempleo, ineficiencia y corrupción) para el desarrollo consistente y continuo de fenotipos complejos, tenidos por competencias, en determinado sistema de formación (educación-capacitación-experiencia).

Los acercamientos parciales al fomento de las competencias de individuos y grupos, pese a contar con bases conceptuales sólidas, pueden incurrir en supuestos falsos que restan impacto, incrementan costos, e incluso, llevan al fracaso a proyectos y programas bajo este enfoque; especialmente, cuando se omiten las particularidades de contextos de formación -de individuos y grupos-, las etapas de madurez cerebral y psicológica, el papel de la experiencia en el desarrollo del cerebro y la neuroplasticidad, y los estadios de las competencias mismas.

\section{REFERENCIAS}

Acarín Tusell, Nolasc y Acarín Pérez-Simó, Laia. (2005). El cerebro del rey. Vida, sexo, conducta, envejecimiento y muerte de los humanos. Barcelona, España: RBA Libros.

Albrecht, Karl. (2008). Inteligencia práctica. El arte y la ciencia del sentido común. Barcelona, España: Javier Vergara Editor.

Biemans, Harm et al. (2004). Competence-based VET in the Netherlands: background and pitfalls. Journal of Vocational Education and Training, 56 (4), 523-538.

Boyatzis, Richard. (1982). The competent manager. A model for effective performance. Nueva York, EE.UU.: Wiley \& Sons. 
Bransford, John; Brown, Ann y Cocking, Rodney (Eds.). (2000). How people learn. Brain, mind, experience, and school. Washington, EE.UU.: National Academy Press.

Climént Bonilla, Juan B. (2009). El papel de las competencias individuales y colectivas en los sistemas de acción. Actualidades Investigativas en Educación, 9 (2), 1-19. Recuperado el 2 de julio de 2010, de http://revista.inie.ucr.ac.cr/articulos/22009/archivos/competencias.pdf

Climént Bonilla, Juan B. (2010). Evaluación de competencias en niños: Un error de apreciación y perspectiva. Actualidades Investigativas en Educación, 10 (3), 1-25. Recuperado el 10 de marzo de 2011, de http://revista.inie.ucr.ac.cr/articulos/32010/archivos/ninios.pdf

Coles, Mike y Werquin, Patrick. (2007). Qualifications systems. Bridges to lifelong learning. París, Francia: OCDE.

Comision Europea. (s.f., a). Elearningeuropa.info. Glosario. Recuperado el 4 de abril de 2009, de www.elearningeuropa.info/main/index.php?lng=es\&page=glossary\&abc=C

Comision Europea. (s.f., b). Elearningeuropa.info. Glossary. Recuperado el 4 de abril de 2009, de www.elearningeuropa.info/main/index.php?page=glossary\&abc=S

Dainty, Andrew; Cheng, Mei-I y Moore, David. (2005). A comparison of the behavioral competencies of client-focused and production-focused project managers in the construction sector. Project Management Journal, 36 (1), 39-48.

Department for Children, Schools and Families (DCSF) and Department for Business, Innovation and Skills (BIS). (s.f.). Functional skills: the facts. Reino Unido. Recuperado el 16 de abril de 2010, de www.dcsf.gov.uk/1419/documents/functional\%20skills\%20policy\%20statement\%20latest.pdf (Consultado 16 de abril de 2010).

Doidge, Norman. (2007). The brain that changes itself. Stories of personal triumph from the frontiers of brain science. Londres, Inglaterra: Penguin Books.

Gardner, Howard. (1993). Multiple intelligences. New horizons in theory and practice. Nueva York, EE.UU.: Basic Books.

Goldberg, Terry y Weinberger, Daniel. (2009). The genetics of cognitive neuroscience. Cambridge, Massachusetts, EE.UU.: MIT Press.

Goleman, Daniel. (1995). Emotional intelligence. Nueva York, EE.UU.: Bantam Books.

Goleman, Daniel. (2006). Social intelligence: The new science of human relationships. Nueva York, EE.UU.: Bantam Books.

Guba, Egon y Lincoln, Yvonna. (1983). Epistemological and methodological bases of naturalistic inquiry. En Madaus, George y Stufflebeam, Daniel (Eds.). Evaluation 
models: Viewpoints on educational and human services evaluation. Massachusetts, EE.UU.: Kluwer-Nijhoff.

Hodkinson, Phil; Sparkes, Andrew y Hodkinson, Heather. (1996). Triumphs and tears: Young people, markets and the transition from school to work. Londres, Inglaterra: David Fulton Publishers.

Hoffmann, Terrence. (1999). The meanings of competency. Journal of European Industrial Training, 23 (6), 275-285.

International Human Genome Sequencing Consortium. (2004). Finishing the euchromatic sequence of the human genome. Nature, 431, 931-945. Recuperado el 1 de junio de 2010, de www.nature.com/nature/journal/v431/n7011/pdf/nature03001.pdf

lowa State University Extension. (2006). Brain development: windows of opportunity. Recuperado el 25 de mayo de 2010, de www.extension.iastate.edu/polk/news/Family+Newsletter.htm

Kandel, Eric; Schwartz, James y Jessell, Thomas (Eds.). (2000). Principles of neural science. Nueva York, EE.UU.: McGraw-Hill.

Kotlyar, Boris. (1992). Plasticity in the nervous system. Washington, EE.UU.: Gordon and Breach Science Publishers.

Lloyd, Chris y Cook, Amanda. (1993). Implementing standards of competence. Practical strategies for industry. Londres, Inglaterra: Kogan Page.

McClelland, David. (1973). Testing for competencies rather than for "intelligence". American Psychologist, 28, 1-14.

Mir, Boris. (2006). Competencias, conocimientos, capacidades y habilidades. La Mirada Pedagógica. Recuperado el 4 de abril de 2009, de www.lamiradapedagogica.blogspot.com/2006/09/competencias-conocimientoscapacidades.html

Mott, Jonathan y Granata, Garin. (2006). The value of teaching and learning technology: Beyond ROI. Educause Quarterly, 29 (2), 48-54.

Nelson, Charles; de Haan, Michelle y Thomas, Kathleen. (2006). Neuroscience of cognitive development. The role of experience and the developing brain. Nueva Jersey, EE.UU.: John Wiley \& Sons.

OCDE. (2002). Definition and selection of competences (DeSeCo). Theoretical and conceptual foundations. Strategy paper. Recuperado el 10 de enero de 2008, de www.portal-stat.admin.ch/deseco/deseco strategy paper final.pdf 
Qualifications and Curriculum Development Agency - QCDA. (2004). Key skills. Standards and guidance 2004. Recuperado el 18 de diciembre de 2009, de www.qcda.gov.uk/6455.aspx

Salovey, Peter y Mayer, John. (1989). Emotional intelligence. Imagination, Cognition and Personality, 9 (3), 185-211.

Smith, Mark. $(2002,2008)$. Howard Gardner and multiple intelligences. The Encyclopedia of Informal Education. Recuperado el 28 de enero de 2011, de http://www.infed.org/thinkers/gardner.htm

Sousa, David. (2006). How the brain learns. California, EE.UU.: Corwin Press.

Spencer, Lyle y Spencer, Signe. (1993). Competence at work. Models for superior performance. Nueva York, EE.UU.: Wiley \& Sons.

Thorndike, Edward. (1920). Intelligence and its use. Harper's Magazine, 140, 227-235.

Woodruffe, Charles. (1993). What is meant by a competency? Leadership and Organization Development Journal, 14 (1), 29-36. 